\title{
Geometric Control Over the Edge Diffraction of Electrically Excited Surface Plasmon Polaritons by Tunnel Junctions
}

Andreea Radulescu, ${ }^{\dagger}$ Vijith Kalathingal, ${ }^{\dagger}$ and Christian A. Nijhuis*

Cite This: ACS Photonics 2021, 8, 3591-3598

Read Online

ABSTRACT: Plasmonic metal-insulator-metal tunnel junctions (MIM-TJs) readily excite surface plasmon polaritons (SPPs) by inelastic electron tunneling, well below the diffraction limit, eliminating the need for bulky optical elements. The highly confined MIM cavity mode (MIM-SPP) excited by the tunneling electrons dominantly outcouples to photons and single-interface SPPs which further outcouple as photons and give characteristic features in the Fourier plane of observation. Here, we employ SPP waveguides, directly integrated with MIM-TJs, to explore the diffraction of singleinterface SPPs at the edges of the metal stripe waveguides. In addition to the leakage of the SPP modes through the metal electrodes, SPP edge diffraction presents as an additional channel for SPP outcoupling, not limited by the thickness of the waveguide. Edge

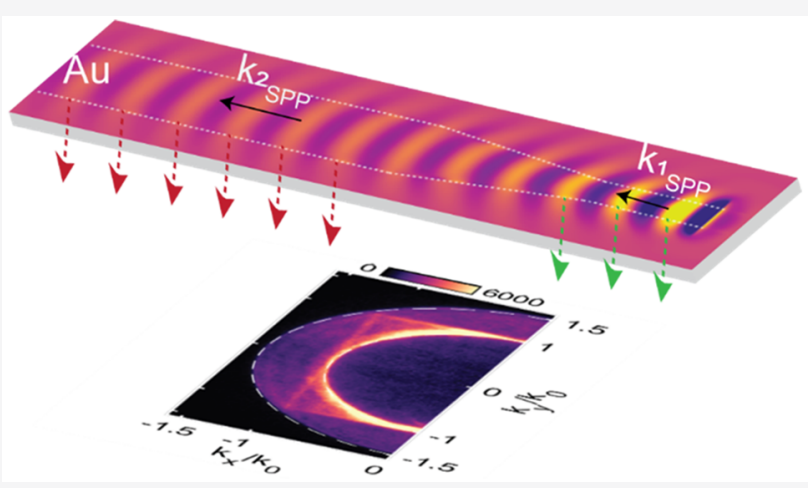
diffraction manifests as a straight-line feature in the Fourier plane of the far-field and by systematically varying the width of the waveguides, we show that the in-plane momentum of the SPPs can be controlled, which in turn leads to control over the edge diffraction. We fabricated MIM-TJs with integrated plasmonic stripe waveguides of different widths, and the propagation and confinement of the SPP modes along the edges of the SPP waveguides are directly identified by the experimental back focal plane imaging, which are further corroborated by numerical simulations. Our findings can be exploited for applications ranging from sensing to nonlinear plasmonics, where focusing and localization of SPP fields are necessary.

KEYWORDS: metal-insulator-metal tunnel junctions, inelastic tunneling, electrical excitation of plasmons, near-field coupling, back focal plane imaging, diffraction

S urface plasmon polaritons (SPPs) have attracted interest in the past years due to their potential in applications such as sensing, ${ }^{1,2}$ plasmonic imaging, ${ }^{3}$ nano-optoelectronics, ${ }^{4-6}$ data transfer and quantum information processing. ${ }^{7-11}$ For these applications, metallic stripe and nanowire based plasmonic waveguides have been used to confine the electromagnetic energy to subwavelength scales. The increased spatial confinement of the SPP modes ${ }^{12-15}$ becomes crucial in nanoscale plasmonic waveguiding, especially when local field enhancements are necessary for strong interactions with quantum emitters ${ }^{16}$ in quantum information processing, or for nonlinear optics, ${ }^{17}$ single-molecule sensing ${ }^{18}$ and ultrafast plasmonic pulse propagation. ${ }^{19}$ For this purpose, on-chip plasmonic focusing ${ }^{20}$ has been demonstrated in planar tapered metallic stripe waveguides, ${ }^{21-24}$ where tapered stripes concentrate the energy of short-range SPPs ${ }^{25}$ beyond the diffraction limit with efficiencies up to $50 \% .^{24,26}$ Moreover, the tapered waveguide geometries were used for second- and third- harmonic generation in the visible wavelength region for plasmonic nanowires of variable widths, demonstrating the potential of SPPs in integrated optical circuits for information processing. ${ }^{27}$

In this context, it is desirable to have access to on-chip electrical sources of SPPs and, for this purpose, plasmonic metal-insulator-metal tunnel junctions (MIM-TJs) are viable candidates. MIM-TJs offer the advantage of on-chip electrical excitation of SPPs by low energy $(<2.5 \mathrm{eV})$ tunneling electrons and, due to their nanoscale footprint, they help to circumvent the diffraction limit associated with optical excitation methods, offering the potential for on-chip device integration. In MIM-TJs (Figure 1a), the energy given off by the inelastically tunneling electrons can couple predominantly to a highly confined cavity mode (MIM-SPP; Figure 1a, red field profile), ${ }^{28}$ which further outcouples to single-interface SPPs (Figure 1a, dark blue and purple field profiles), photons (Figure 1a, green arrows), and thermal loss. However, in the context of SPP waveguiding, the geometry (width and thickness) and the surface discontinuities of the plasmonic waveguides $^{13,29,30}$ integrated directly to the MIM-TJs play an

Received: August 2, 2021

Published: November 12, 2021 


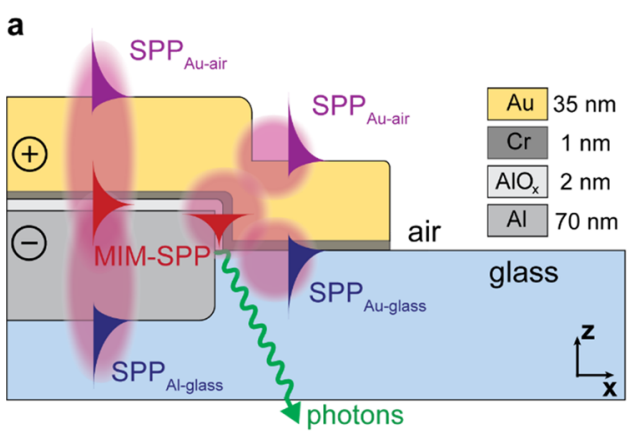

b

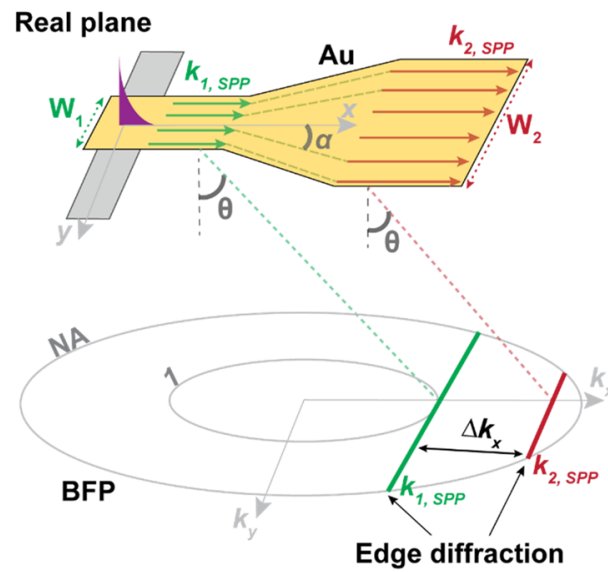

Figure 1. (a) Schematic showing the outcoupling of the MIM-SPP (red) mode to metal-air (purple) and metal-glass (dark blue) SPP modes and photons (green arrow). (b) Schematic showing the momentum matching conditions for the edge diffraction of the leaky SPP modes (metal-air SPP) into the glass substrate (top). The inplane SPP momentum increases from $k_{1, \mathrm{SPP}}$ (green arrows) to $k_{2, \mathrm{SPP}}$ (red arrows) by $\Delta k_{x}$ (not to scale) with the change in width of the plasmonic waveguide from $W_{1}$ to $W_{2}$. The angle $\alpha$ represents the tapering angle for the waveguide and $\theta$ is the photon emission angle into the glass substrate $\left(\theta=\sin ^{-1}\left(k_{\mathrm{SPP}} / n k_{0}\right)\right.$ for $\varphi=0$ from eq 1 , where $\varphi$ is the azimuthal angle in the BFP (bottom). The inner circle of the BFP at $k / k_{0}=1$ corresponds to the critical angle at the glassair interface, and the outer circle represents the numerical aperture (NA) of the objective.

important role in dictating the confinement and the propagation length of the guided SPP modes. Hence, to properly design plasmonic devices, it is important to understand how the outcoupled SPPs are confined along and interact with the edges of the waveguides.

For plasmonic waveguides of finite widths, SPP leakage and scattering is considered as the main outcoupling or decay channel. ${ }^{31}$ However, as demonstrated in previous works, the edges of the plasmonic waveguide affect the lateral confinement of the SPPs as well as their propagation length. ${ }^{29,32,33}$ As the width $(W)$ of the waveguides decreases, the interaction of the SPPs with the edges of the waveguides becomes more pronounced, contributing to the decaying propagation length of SPPs in narrow metallic stripes. ${ }^{34,35}$ More importantly, the angle at which SPPs travel with respect to the waveguide becomes crucial in the outcoupling process of SPPs upon interaction with the waveguide edges. When the SPPs propagate normal to the edges, they mainly undergo backreflection or outcouple via scattering. ${ }^{33}$ But, when the SPPs travel along the edges, they undergo diffraction at the edges of the waveguide, which provides an additional outcoupling channel for the SPPs as photons, provided the momentum mismatch between SPPs and photons is compensated. ${ }^{35,36}$ For instance, in the case of a metallic stripe waveguide with glass and air as the surrounding dielectric media, the edge diffraction has been observed for SPPs excited at the metal-air interface, $^{35}$ given that the momentum $\left(k_{\mathrm{SPP}}\right)$ of the metalair SPPs is lower than that of the photons in the substrate (glass) $)^{31,37}$ and can outcouple according to the momentum conservation equation (eq 1 ):

$$
k_{\mathrm{SPP}}=n k_{0} \sin \theta \cos \varphi
$$

where $n$ is the refractive index of the glass substrate, $k_{0}$ is the free-space wavenumber of light, $\theta$ is the emission angle into the substrate, and $\varphi$ is the azimuthal angle in the back focal plane (BFP) projection corresponding to the in-plane propagation direction. Therefore, the edges of the waveguide allow a directional SPP leakage to occur, ${ }^{35}$ creating an additional outcoupling pathway that is not screened by the thickness of the metallic waveguide.

Recently, for MIM-TJs with integrated SPP stripe waveguides, various SPP outcoupling pathways were identified, ${ }^{38}$ control over the outcoupling pathways was demonstrated by controlling the tunneling direction, ${ }^{39}$ and plasmonic antennas were used to enhance the SPP outcoupling. ${ }^{40}$ It was also demonstrated that the outcoupling pathways can be selectively turned on and off by changing the electrode thickness. ${ }^{38}$ In these studies, BFP imaging of the far-field light emission serves as a powerful tool to identify various outcoupling channels for the MIM-SPP and SPPs when they undergo roughnessinduced scattering, ${ }^{41}$ leakage, or scattering at the waveguide discontinuities. However, the edge diffraction of SPPs, which provides a complementary way of far-field SPP characterization, as it can readily be identified through BFP imaging as a straight-line feature adjacent to the leakage of the propagating SPPs $\left(k_{\mathrm{SPP}}\right),{ }^{35,36,42}$ has not been examined in detail before. Compared to optical excitation schemes, where the position of the plasmonic waveguide within the laser waist beam has to be precisely controlled in order to excite SPPs close to the edges of the waveguide, ${ }^{30,35,42-44}$ in MIM-TJs connected to plasmonic waveguides, SPPs can easily be excited across the entire width of the waveguides and the edge diffraction can easily be obtained as previously observed. ${ }^{38,39,41,45}$ Additionally, the edge diffraction clearly separates the metal-air leaky SPP contributions from those of the metal-glass SPPs, which can only be detected via scattering at the end of the waveguide due to the bound nature of the modes. ${ }^{38,46}$ Hence, an integrated structure where the MIM-TJ is in-plane with a plasmonic stripe waveguide offers an efficient platform for investigating the SPP edge diffraction. Moreover, even though the interaction of SPPs with the edges has been discussed in previous works, ${ }^{35,36}$ no control over the edge diffraction process has been demonstrated so far.

Here we experimentally demonstrate the edge diffraction of SPPs in plasmonic stripe waveguides, directly integrated with MIM-TJs. The plasmonic waveguides support both metal-air (leaky) and metal-glass (bound) modes (Figure 1a) and the edge diffraction provides an additional way to characterize the SPP outcoupling in the far-field measurements. We also demonstrate control over the edge diffraction through the control of the SPP in-plane momentum, by changing the width $(W)$ of the waveguides (Figure $1 \mathrm{~b}$ ), which manifests as a shift in the position of the straight-line feature in the BFP corresponding to the edge diffraction. We further design a 

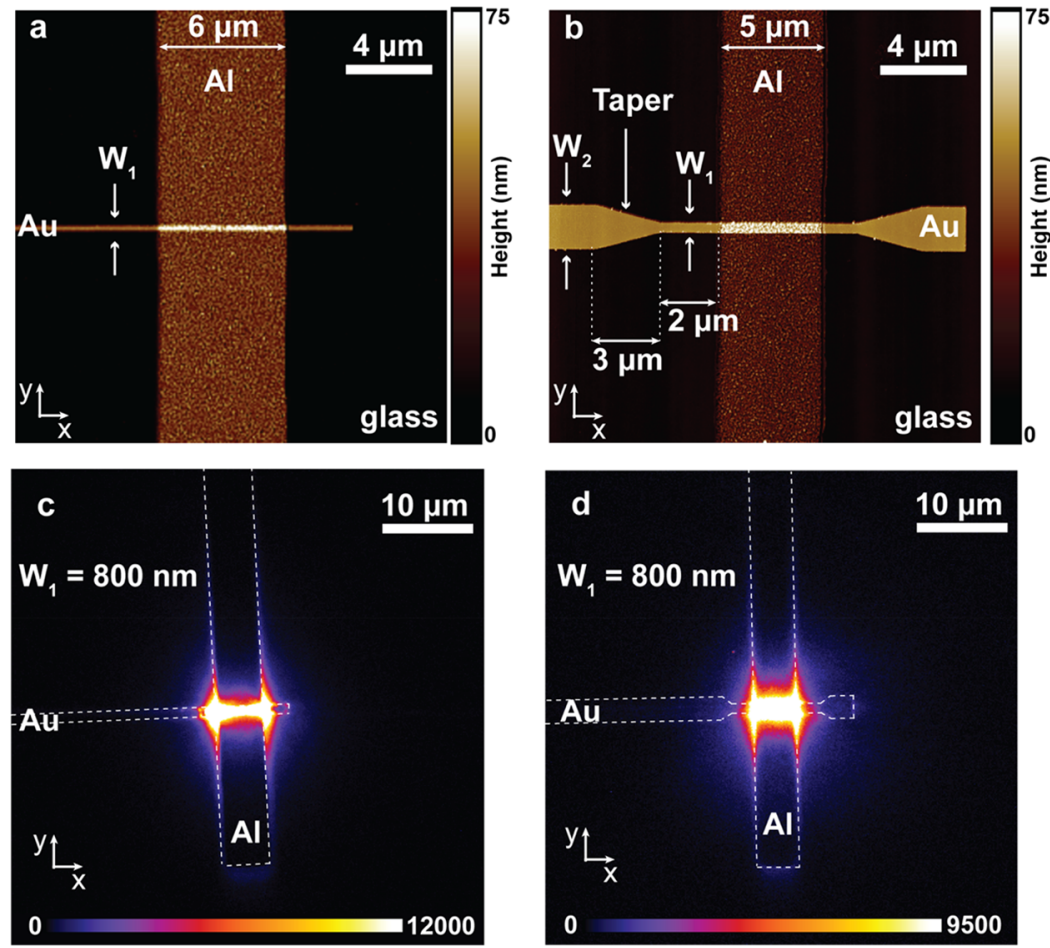

Figure 2. Representative AFM images of the MIM-TJ and associated (a) linear $\left(W_{1}=600 \mathrm{~nm}\right)$ and (b) tapered Au stripe waveguides $\left(W_{1}=800\right.$ $\mathrm{nm}$ and $\left.W_{2}=2 \mu \mathrm{m}\right)$. The Au waveguides are $35 \mathrm{~nm}$ thick, and the Al stripe has a fixed length $(20 \mu \mathrm{m})$, width $(5-6 \mu \mathrm{m})$, and thickness $70 \mathrm{~nm}$. (c, d) Real plane images of the light emission of representative devices with $W_{1}=800 \mathrm{~nm}$ for (c) linear and (d) tapered waveguides, respectively. The images are recorded at a voltage of $-1.6 \mathrm{~V}$ and the dashed lines are visual guides, indicating the electrodes.

tapered plasmonic stripe waveguide that comprises of two segments with different widths connected by a tapered section (Figure 1b) to further demonstrate the change in the in-plane momentum of the excited metal-air SPPs as they propagate along the tapered region. We use far-field optical microscopy, namely BFP imaging which gives the angular distribution of the emitted radiation propagating in a specific direction, as an active tool to measure the $k$-vector of the excited SPPs and to determine the change of the in-plane momentum (marked as $\Delta k_{x}$ in Figure $1 \mathrm{~b}$ ). The experimental observations are then supported by numerical simulations using the finite-difference time-domain (FDTD) method.

\section{EXPERIMENTAL DESIGN}

To describe the in-plane momentum contributions, we look at the in-plane components of $k_{\mathrm{SPP}}$, as shown in eq 2 :

$$
k_{\text {SPP }}^{2}=k_{x}^{2}+k_{y}^{2}
$$

Each $k_{\mathrm{SPP}}$ in-plane component $\left(k_{x}\right.$ and $\left.k_{y}\right)$ has a Fourier uncertainty associated with it, where each uncertainty in position $\Delta x$ or $\Delta y$ is related to the uncertainty in momentum $\left(\Delta k_{x}\right.$ or $\left.\Delta k_{y}\right)$ by $^{47}$

$$
\begin{aligned}
& \Delta k_{x} \Delta x \geq 2 \pi \\
& \Delta k_{y} \Delta y \geq 2 \pi
\end{aligned}
$$

Based on the equations shown above, we designed the tapered plasmonic waveguide whose dimensions in the $y$ direction change from $W_{1}$ to $W_{2}$ and are connected by a tapered transition section (Figure $1 \mathrm{~b}$ ), providing a change in $\Delta y$ (in terms of $\alpha$ ). According to eq 4 , an increase in $\Delta y$ leads to a decrease in $\Delta k_{y}$, which effectively increases $\Delta k_{x}$ due to the total momentum conservation given by eq 2 and can be observed as the shift of the straight-line feature corresponding to $k_{x}$ in the Fourier plane.

For this work, we used $\mathrm{Al}-\mathrm{AlO}_{x}-\mathrm{Au}$ MIM-TJs as SPP excitation sources due to their broadband nature ${ }^{38,46}$ and because they are well-characterized in literature. ${ }^{40,48}$ The devices were fabricated on borosilicate glass coverslips through electron-beam lithography patterning, thermal evaporation of the $\mathrm{Al}$ electrode, TMAH (tetramethylammonium hydroxide) etching of $\mathrm{Al}$ and native oxidation in air for the growth of the $\mathrm{AlO}_{x}$ tunnel barrier ( $\sim 2 \mathrm{~nm}$ thick) and electron beam evaporation of $\mathrm{Au}$, with $1 \mathrm{~nm}$ of $\mathrm{Cr}$ as adhesion layer (see Supporting Information, section S1 for the fabrication details). Figure 2a,b shows characteristic AFM images of the devices used in this study. The $\mathrm{Au}$ electrode, which acts as the plasmonic waveguide, has a thickness of $35 \pm 2 \mathrm{~nm}$. We chose this thickness to allow not only the excitation of SPPs at the $\mathrm{Au}$-glass interface $\left(\mathrm{SPP}_{\text {Au-glass }}\right)$, but also the excitation of SPPs at the $\mathrm{Au}$-air interface $\left(\mathrm{SPP}_{\mathrm{Au} \text {-air }}\right),{ }^{38}$ which undergo the edge diffraction process described above. The $\mathrm{Al}(70 \pm 6 \mathrm{~nm}$ thickness) constitutes the bottom electrode and is partially embedded inside the glass substrate $(40 \pm 3 \mathrm{~nm}$ sunken into the substrate, $30 \pm 3 \mathrm{~nm}$ above the glass substrate). We chose this design to ensure that the top Au electrode is continuous as it lays over the thicker $\mathrm{Al}$ electrode. We further extracted the root-mean-squared surface roughness, which is $7 \pm 3 \mathrm{~nm}$ and 1 $\pm 0.2 \mathrm{~nm}$ for $\mathrm{Al}$ and $\mathrm{Au}$, respectively, both measured on the waveguides extending away from the MIM-TJ area.

Figure $2 \mathrm{a}$ shows the design of the linear metallic stripe waveguides for which the width was changed $\left(W_{1}=600 \mathrm{~nm}\right.$, $800 \mathrm{~nm}, 1 \mu \mathrm{m}$, and $2 \mu \mathrm{m}$ ), whereas Figure $2 \mathrm{~b}$ shows the design of the tapered $\mathrm{Au}$ waveguides used in this study, where two segments of different widths $\left(W_{1}\right.$ and $\left.W_{2}\right)$ are connected by a 

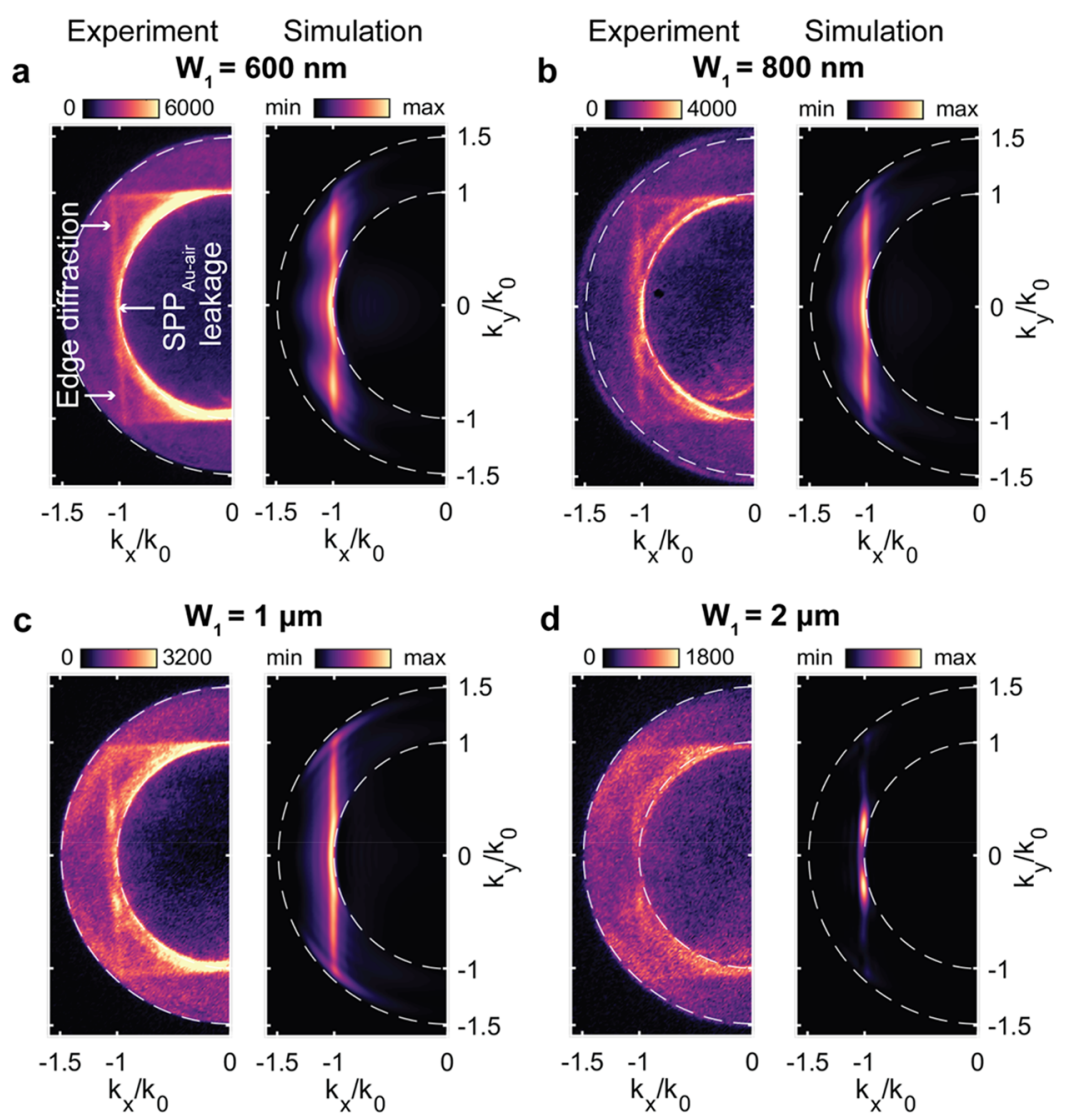

Figure 3. Experimental and simulated BFP images of the light emission from the MIM-TJs with linear waveguides, for $W_{1}=600 \mathrm{~nm}(\mathrm{a}), 800 \mathrm{~nm}$ (b), $1 \mu \mathrm{m}(\mathrm{c})$, and $2 \mu \mathrm{m}(\mathrm{d})$. All images are collected at $-1.6 \mathrm{~V}$. The left panels show the experimental BFP images, and the right panels show the FDTD simulations. The Fourier axes are restricted to the negative side of $k_{x} / k_{0}(-1.5$ to 0$)$, as the edge diffraction appears in this range. The inner dashed white circle represents the $k / k_{0}=1$, which corresponds to the critical angle at the glass-air interface, and the outer dashed white circle represents the NA of the objective, with $k / k_{0}=1.49$.

tapered section. For our work we chose $W_{1}=600$ or $800 \mathrm{~nm}$ and $W_{2}=2 \mu \mathrm{m}$ to alter the in-plane momentum of the SPPs.

Prior to the optical characterization, we recorded the $I(V)$ curves (see Supporting Information, section S2) of the MIMTJs using two Signatone micromanipulators with tungsten probes and a source meter (Keithley 6430) controlled by a homemade LabView program. As explained in ref 49 (and in more detail in refs 50 and 51), tunnel junctions break down due to the formation of metal filaments caused by the wind force (direct momentum transfer between the conduction electrons and metal atoms) or Joule heating, which may be bias polarity dependent when different electrode materials are used. Our junctions are more stable when a negative bias is applied to the $\mathrm{Al}$ electrode than vice versa. For these reasons we limited the bias range to $-1.6 \mathrm{~V}$ to avoid electrical breakdown of our junctions. The $I(V)$ curves show an exponential behavior, which is in accordance with previously reported MIM-TJ devices. ${ }^{38,40,46}$

For the far-field optical measurements, we recorded the real plane and BFP images of the light emission from the MIM-TJs in an inverted optical microscope equipped with an electron multiplying charge-coupled device (EMCCD) camera and a Bertrand lens (which enables the projection of the BFP onto the EMCCD). The light emission is collected through the glass substrate using a $100 \times$ oil immersion objective with an NA of 1.49. Figure $2 \mathrm{c}, \mathrm{d}$ shows the real plane image of two representative devices, for the linear waveguide $\left(W_{1}=800\right.$ $\mathrm{nm}$; Figure 2c) and for the tapered waveguide $\left(W_{1}=800 \mathrm{~nm}\right.$;
Figure 2d), at $-1.6 \mathrm{~V}$ applied bias. To account for the low quantum efficiency of the light emission (see Supporting Information, section S3 for spectra and quantum efficiency plot), the images were recorded with an integration time of 2 min and an EM gain of 300 .

\section{RESULTS AND DISCUSSION}

To investigate the change of the SPP in-plane momentum, we recorded the BFP images of the devices described in Figure 2. As mentioned in the first section of this paper, the edge diffraction of SPPs is evidenced as a straight-line feature in the $\mathrm{BFP}^{36}$ of the far-field light emission from the devices. This can be understood through eqs $2-4$ as follows: when the SPPs (with in-plane momentum $k_{\mathrm{SPP}}$ ) are propagating along one direction, for instance, along the $x$-direction in Figure $1 \mathrm{~b}$, the spatial confinement in $y(\Delta y \approx 0)$ will force $\Delta k_{y}$ to span the complete range of $k_{y}\left(-k_{y}\right.$ to $\left.+k_{y}\right)$ according to the Fourier uncertainty (eq 4). For SPPs traveling along the edges of the waveguide, the diffraction at the edges then results in an effective SPP leakage, preserving the original in-plane momentum $\left(k_{\mathrm{SPP}}\right)$ and appears as a narrow line at $k_{x} / k_{0} \sim 1$ (adjacent to $k_{\mathrm{SPP}} / k_{0}$ circle in the BFP), which spans the entire $k_{y} / k_{0}$ range.

In the left panels of Figure 3 we show the experimental BFP images of the devices with $W_{1}=600 \mathrm{~nm}$ (Figure 3a), $800 \mathrm{~nm}$ (Figure $3 \mathrm{~b}$ ), $1 \mu \mathrm{m}$ (Figure $3 \mathrm{c}$ ), and $2 \mu \mathrm{m}$ (Figure $3 \mathrm{~d}$ ). The Fourier axes are restricted to the negative $k_{x} / k_{0}(-1.5$ to 0$)$, as the straight-line feature corresponding to the edge diffraction 
appears in this range (see Supporting Information, section S3, for the full data set). We also indicate the critical angle at the glass-air interface as the inner dashed circle at $k / k_{0}=1$, and the NA of the objective as the outer dashed circle at $k / k_{0}=$ 1.49. We observe a vertical straight-line feature close to $k_{x} / k_{0}=$ -1 , which corresponds to the edge diffraction of the $\mathrm{SPP}_{\mathrm{Au} \text {-air }}$ Also, for all images, we observe horizontal straight lines close to $\left|k_{y} / k_{0}\right|=1$, which correspond to the edge diffraction of $\mathrm{SPP}_{\mathrm{Al} \text {-air }}$ modes that are excited by the MIM-TJ. It should be noted that the intensities of the $\mathrm{Au}$-air and $\mathrm{Al}-$ air SPP edge diffraction are comparable, even though $\mathrm{Al}$ is more lossy compared to Au. This is further discussed in the context of thickness dependence of the edge diffraction below. To demonstrate the control of the edge diffraction mechanism, we focus on the edge diffraction of the $\mathrm{SPP}_{\mathrm{Au} \text {-air }}$ for which we designed the tapered waveguide described in Figure $1 \mathrm{~b}$.

Figure 4 shows the cross-section intensity profiles extracted for the straight-line features in the BFP images (Figure $3 a-c$ ). As the $2 \mu \mathrm{m}$ waveguide does not show explicit straight-line
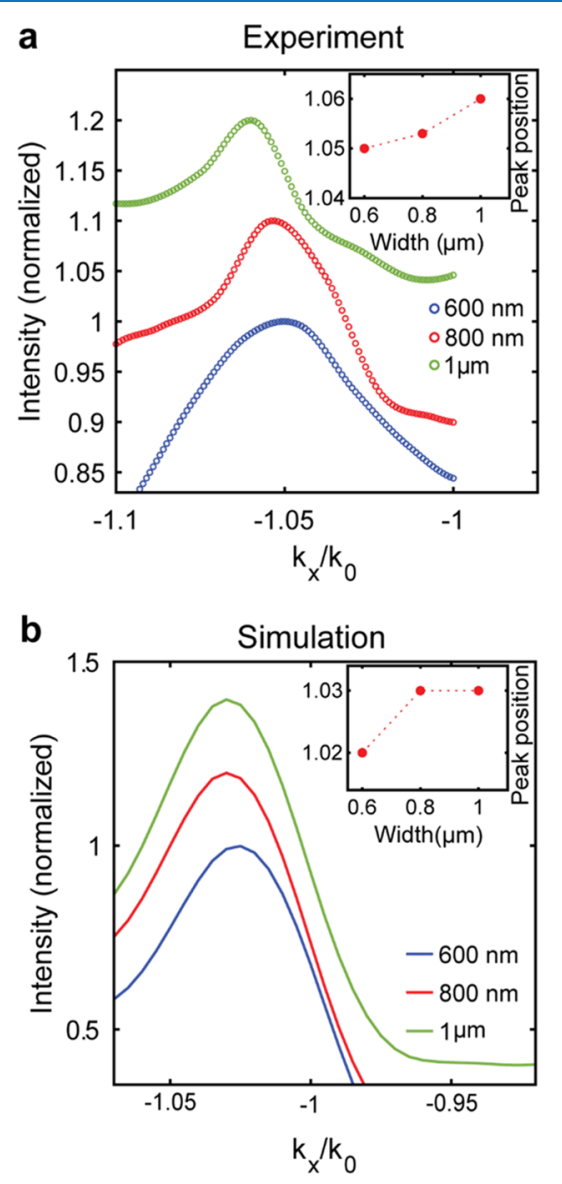

Figure 4. (a) Intensity line profile for the straight-line features in Figure $3 \mathrm{a}-\mathrm{c}$, oriented along the $k_{y}$ axis, corresponding to $W_{1}=600$ $\mathrm{nm}, 800 \mathrm{~nm}$, and $1 \mu \mathrm{m}$. For the experimental BFP images (left panels in Figure $3 \mathrm{a}-\mathrm{c}$ ), the line profiles are calculated by averaging the intensity along $-1.1<k_{x}<-1$ for all values of $k_{y}$ between \pm 0.5 to \pm 0.9 to avoid the contributions from the leakage and scattering close to $k_{y} \sim 0$. The inset shows the peak position of the straight-lines features (in terms of $\left.\left|k_{x} / k_{0}\right|\right)$ as a function of $W_{1}$. (b) Line profile extracted from the FDTD results shown in the right panels of Figure $3 a-c$ and the inset shows the evolution of the peak positions. In both (a) and (b), the line profiles are shifted vertically to enhance the visibility and the dotted lines in the insets are guide to the eye. features in the BFP, we restrict the analysis to $W_{1}=600 \mathrm{~nm}$, $800 \mathrm{~nm}$, and $1 \mu \mathrm{m}$ cases. For the experimental BFP images (left panels in Figure $3 \mathrm{a}-\mathrm{c}$ ), the line profiles are obtained by averaging the intensity along $-1.1<k_{x}<-1$ for all values of $k_{y}$ between \pm 0.5 to \pm 0.9 to avoid the contributions from the strong leakage and scattering close to $k_{y} \sim 0$ and are plotted in Figure $4 \mathrm{a}$. The inset shows the peak positions obtained from the line profiles and corresponds to the evolution of the effective index of the SPP modes (straight-lines features in Figure 3) as a function of $W_{1}$.

From the above BFP images (Figure 3) and intensity line profiles (Figure 4), we observe the following: First, the position of the straight-line feature close to $k_{x} / k_{0}=-1$ shifts on the $k_{x}$ axis as $W_{1}$ increases. As the width increases from 600 $\mathrm{nm}$ to $1 \mu \mathrm{m}$, the peak position in the line profile shifts from 1.05 to 1.06 (in terms of $\left|k_{x} / k_{0}\right|$ ), which indicates an increase in the effective index of the SPP mode supported by the metal stripe. Second, the leakage contribution of the $\mathrm{SPP}_{\mathrm{Au} \text {-air }}$ mode becomes more prominent compared to the edge diffraction as $W_{1}$ increases. The first observation can be explained by the change of in-plane momentum of the SPPs, discussed in eqs $2-4$. As $W_{1}$ increases (along the $y$ direction; Figure $1 \mathrm{~b}$ ), the confinement of SPPs along the waveguide edges decreases, leading to a decrease in the $k_{y}$ in-plane momentum component. Due to the total momentum conservation, this leads to an increase in the $k_{x}$ component, hence, the position of the straight-line in the BFP shifts to higher $\left|k_{x} / k_{0}\right|$ values. Here, however, it should be noted that the emission from the junction is still broadband, not affected by the change in the width of the electrode (see Supporting Information, section S3 and Figure S4, for the spectra).

The second observation can be explained through the change in the spread of the SPP momentum $\left(\Delta k_{\mathrm{SPP}}\right)$ versus $W_{1}$. When $W_{1}$ becomes smaller than a particular limit (in this work, $<600 \mathrm{~nm}$ ), the effective index of the supported SPP modes becomes comparable to the index of the medium, in this case air $\left(n_{\text {eff }}<1\right)$, and the modes are radiative in nature. ${ }^{30}$ However, at $W_{1}>2 \mu \mathrm{m}$, the SPP mode propagation transforms from linear, along the waveguide edges, to radial across the width of the waveguide, starting from the MIM-TJ area. This leads to a finite spread in the in-plane SPP momentum $\left(\Delta k_{\mathrm{SPP}}\right)$, with the leakage contribution detected as an arc (close to $k / k_{0}=1$ ) in the Fourier plane, which is more prominent than the straight-line contribution from the edge diffraction. Also, for larger widths, the in-plane momentum of the SPP mode saturates to the SPP mode-index of the Au-air interface of a semi-infinite Au thin film. ${ }^{31}$

To investigate the momentum distribution of the SPP edge diffraction from the stripe waveguides, we performed threedimensional FDTD simulations using Lumerical. ${ }^{52}$ The fundamental SPP mode supported by the stripe waveguide for a given width is calculated in the initial step and then used as the excitation source for the MIM-TJ. Typically, inelastic tunneling in MIM-TJs is represented by a dipole source with a broadband spectral response and leads to the excitation of all the supported modes by the metal stripes (with different coupling efficiency) due to the near-field character of the dipole excitation. Here, to simplify the analysis, we restrict the excitation to the fundamental SPP mode and investigate the waveguiding and the far-field outcoupling from the metal stripes. A near-to-far-field transformation of the SPP near-field is used to calculate the time-averaged field intensity in the farfield (right panels of Figure 3), which gives the BFP images 
(see Supporting Information, section S4). Figure 4b shows the line profiles extracted from the FDTD simulation results shown in the right panels of Figure $3 a-c$, and the inset gives the peak positions obtained from the line profiles. The peak evolution from the experiments is in qualitative agreement with simulations, as the increase in the width of the waveguide follows with a corresponding increase in the effective index. In both Figure $4 a$ and $b$, the line profiles are shifted vertically to enhance the visibility, and the dotted lines in the insets are a guide to the eye.

We further discuss the MIM-TJs with tapered waveguides. Figure 5a shows the experimental BFP images for $W_{1}=600$ $\mathrm{nm}$ (left) and $800 \mathrm{~nm}$ (right) and for $W_{2}=2 \mu \mathrm{m}$. From these images, we clearly observe that, as the width changes from $W_{1}$ to $W_{2}$, the position of the edge diffraction changes in the BFP. Moreover, we also observe a tilted feature with continuous
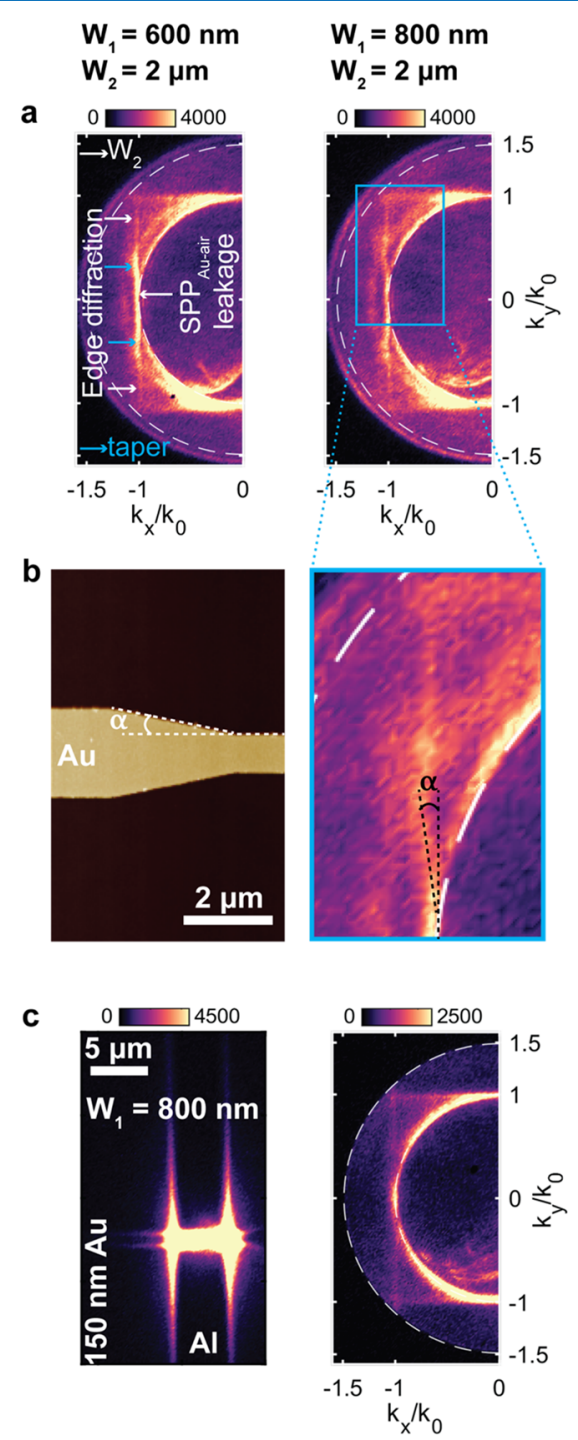

Figure 5. (a) Experimental BFP images for the MIM-TJs with tapered waveguides, for $W_{1}=600 \mathrm{~nm}$ (left) and $800 \mathrm{~nm}$ (right). All images are collected at $-1.6 \mathrm{~V}$. (b) AFM image showing the taper angle, $\alpha$, (left) and zoomed-in BFP image (according to the blue box in the right panel of (a)) showing the contribution of the tapered region onto the BFP (right), for a tapered waveguide with $W_{1}=800 \mathrm{~nm}$. (c) Real plane (left) and BFP (right) images of a MIM-TJ with a waveguide of $150 \mathrm{~nm}$ Au thickness, showing edge diffraction. change in $k_{x}$ as the SPPs propagate from $W_{1}$ to the $W_{2}$ across the tapered segment. The angle of the tilted feature, $\alpha$ (shown in the right panel of Figure $5 b$ ), follows the angle of the tapered section (shown in the AFM image, left panel of Figure $5 b)$. Hence, we attribute the tilted feature to the edge diffraction of SPPs at the edges of the tapered segment. However, it should be noted that, as compared to the SPP excitation with a monochromatic source (for example, in ref 35), where sharp features are observed in the BFP, the broadband nature of the MIM-TJ excitation results in broad features that are relatively difficult to isolate in the BFP analysis.

Lastly, we discuss the edge diffraction in the case of a metallic stripe waveguide with a thickness much higher than the skin depth of Au. ${ }^{53}$ Figure $5 \mathrm{c}$ shows the real plane (Figure $5 \mathrm{c}$, left) and the BFP (Figure 5c, right) images of the light emission of a MIM-TJ connected to a linear Au waveguide, with $W_{1}=800 \mathrm{~nm}$ and a thickness of $150 \mathrm{~nm}$. As mentioned in the first section of the paper, the edge diffraction leakage mechanism is not screened by the metallic electrode, hence, the signature of this outcoupling mechanism can still be observed for thick waveguides. In the real-plane image, we observe an increased light intensity along the edges of the waveguide, and in the BFP image, we clearly see the straightline feature, confirming the assignment of the straight-line to the edge diffraction mechanism. Also, both $\mathrm{Au}-$ air and $\mathrm{Al}-$ air give comparable intensities for the edge diffraction in the BFP images, as shown in Figure 3. This observation confirms the fact that the edge diffraction of the metal-air SPP modes is least affected by the electromagnetic screening.

\section{CONCLUSIONS}

The study presented here shows that the edge diffraction constitutes an additional outcoupling mechanism for the SPPs propagating along plasmonic waveguides and interacting with their edges. By systematically varying the width of the waveguides, we show that we can control the in-plane momentum of SPPs, which in turn leads to control over the edge diffraction. Through BFP imaging and FDTD simulations, we identify the signature of the edge diffraction as a straight-line feature in the far-field, which shifts in position as the width of the waveguide increases. Furthermore, by using tapered waveguides, we show that the edge diffraction follows the edge of the tapered segment, leading to a tilted feature in the BFP, with continuously changing $k_{x}$ value. Lastly, we confirm that the straight-line in the BFP is caused by edge diffraction by investigating a Au waveguide of $150 \mathrm{~nm}$ thickness and show that the edge diffraction is least affected by the electromagnetic screening of the metal electrodes. This provides an opportunity to optimize the desired shift in momentum for the SPP modes by using the width and thickness of the waveguide as variable parameters, by reducing the leakage of the modes as photons. This can be further optimized for the SPP focusing by geometrically tuning the effective index of the SPP waveguide (by varying the crosssection profile, width, and thickness) directly coupled to the source (MIM-TJ), exploiting the versatility of the electrical excitation of SPPs from TJs. The $k$-vector of the SPP edge diffraction is also directly correlated with the index of the surrounding dielectric medium, which can be replaced by a host medium for applications related to sensing. These results can guide the design of future SPP devices for applications in sensing, quantum information processing and nonlinear optics 
where the focusing and localization of SPP fields at the edges of the waveguides could provide the necessary field enhancement for single-molecule detection and interactions with quantum emitters.

\section{ASSOCIATED CONTENT}

\section{SI Supporting Information}

The Supporting Information is available free of charge at https://pubs.acs.org/doi/10.1021/acsphotonics.1c01173.

Device fabrication; Electrical measurements; Optical characterization; Numerical simulation details (PDF)

\section{AUTHOR INFORMATION}

\section{Corresponding Author}

Christian A. Nijhuis - Integrative Sciences and Engineering Programme, NUS Graduate School, National University of Singapore, Singapore 119077, Singapore; Centre for Advanced 2D Materials and Graphene Research Centre, National University of Singapore, Singapore 117564, Singapore; Department of Chemistry, National University of Singapore, Singapore 117543, Singapore; Hybrid Materials for Optoelectronics Group, Department of Molecules and Materials, MESA+ Institute for Nanotechnology and Center for Brain-Inspired Nano Systems (BRAINS), Faculty of Science and Technology, University of Twente, 7500 AE Enschede, The Netherlands; 이이.org/0000-0003-34354600; Email: c.a.nijhuis@utwente.nl

\section{Authors}

Andreea Radulescu - Integrative Sciences and Engineering Programme, NUS Graduate School, National University of Singapore, Singapore 119077, Singapore; Centre for Advanced 2D Materials and Graphene Research Centre, National University of Singapore, Singapore 117564, Singapore; Department of Chemistry, National University of Singapore, Singapore 117543, Singapore

Vijith Kalathingal - Centre for Advanced 2D Materials and Graphene Research Centre, National University of Singapore, Singapore 117564, Singapore; Department of Chemistry, National University of Singapore, Singapore 117543, Singapore; Department of Electrical and Computer Engineering, National University of Singapore, Singapore 117576, Singapore; 이이이.org/0000-0002-4003-9765

Complete contact information is available at:

https://pubs.acs.org/10.1021/acsphotonics.1c01173

\section{Author Contributions \\ ${ }^{\dagger}$ A.R. and V.K. contributed equally to this work.}

\section{Notes}

The authors declare no competing financial interest.

\section{ACKNOWLEDGMENTS}

The authors acknowledge the National Research Foundation (NRF) for supporting this research under the Prime Minister's Office, Singapore, under its Medium Sized Centre Programme and the Competitive Research Programme (CRP; NRFCRP17-2017-08). The authors also thank the Centre for Advanced 2D Materials (CA2DM) for the provided facilities.

\section{REFERENCES}

(1) Yu, H.; Shan, X.; Wang, S.; Chen, H.; Tao, N. Plasmonic imaging and detection of single DNA molecules. ACS Nano 2014, 8 (4), 3427-33.

(2) Lopez, G. A.; Estevez, M. C.; Soler, M.; Lechuga, L. M. Recent advances in nanoplasmonic biosensors: applications and lab-on-a-chip integration. Nanophotonics 2017, 6 (1), 123-136.

(3) Yang, Y.; Shen, G.; Wang, H.; Li, H.; Zhang, T.; Tao, N.; Ding, $\mathrm{X}$.; Yu, H. Interferometric plasmonic imaging and detection of single exosomes. Proc. Natl. Acad. Sci. U. S. A. 2018, 115 (41), 10275.

(4) Sorger, V. J.; Oulton, R. F.; Ma, R.-M.; Zhang, X. Toward integrated plasmonic circuits. MRS Bull. 2012, 37 (8), 728-738.

(5) Tuniz, A.; Bickerton, O.; Diaz, F. J.; Kasebier, T.; Kley, E. B.; Kroker, S.; Palomba, S.; de Sterke, C. M. Modular nonlinear hybrid plasmonic circuit. Nat. Commun. 2020, 11 (1), 2413.

(6) Karabchevsky, A.; Katiyi, A.; Ang, A. S.; Hazan, A. On-chip nanophotonics and future challenges. Nanophotonics 2020, 9 (12), 3733-3753.

(7) O'Brien, J.; Patton, B.; Sasaki, M.; Vučković, J. Focus on integrated quantum optics. New J. Phys. 2013, 15 (3), 035016.

(8) de Leon, N. P.; Lukin, M. D.; Park, H. Quantum Plasmonic Circuits. IEEE J. Sel. Top. Quantum Electron. 2012, 18 (6), 17811791.

(9) Wei, H.; Wang, Z.; Tian, X.; Käll, M.; Xu, H. Cascaded logic gates in nanophotonic plasmon networks. Nat. Commun. 2011, 2 (1), 387.

(10) Chang, D. E.; Sorensen, A. S.; Hemmer, P. R.; Lukin, M. D. Quantum optics with surface plasmons. Phys. Rev. Lett. 2006, 97 (5), 053002 .

(11) Gonzalez-Tudela, A.; Martin-Cano, D.; Moreno, E.; MartinMoreno, L.; Tejedor, C.; Garcia-Vidal, F. J. Entanglement of two qubits mediated by one-dimensional plasmonic waveguides. Phys. Rev. Lett. 2011, 106 (2), 020501.

(12) Bozhevolnyi, S. I.; Volkov, V. S.; Devaux, E.; Ebbesen, T. W. Channel plasmon-polariton guiding by subwavelength metal grooves. Phys. Rev. Lett. 2005, 95 (4), 046802.

(13) Moreno, E.; Rodrigo, S. G.; Bozhevolnyi, S. I.; Martin-Moreno, L.; Garcia-Vidal, F. J. Guiding and focusing of electromagnetic fields with wedge plasmon polaritons. Phys. Rev. Lett. 2008, 100 (2), 023901.

(14) Oulton, R. F.; Sorger, V. J.; Genov, D. A.; Pile, D. F. P.; Zhang, $\mathrm{X}$. A hybrid plasmonic waveguide for subwavelength confinement and long-range propagation. Nat. Photonics 2008, 2 (8), 496-500.

(15) Sorger, V. J.; Ye, Z.; Oulton, R. F.; Wang, Y.; Bartal, G.; Yin, X.; Zhang, X. Experimental demonstration of low-loss optical waveguiding at deep sub-wavelength scales. Nat. Commun. 2011, 2 (1), 15.

(16) Akimov, A. V.; Mukherjee, A.; Yu, C. L.; Chang, D. E.; Zibrov, A. S.; Hemmer, P. R.; Park, H.; Lukin, M. D. Generation of single optical plasmons in metallic nanowires coupled to quantum dots. Nature 2007, 450 (7168), 402-6.

(17) Bouhelier, A.; Beversluis, M.; Hartschuh, A.; Novotny, L. Nearfield second-harmonic generation induced by local field enhancement. Phys. Rev. Lett. 2003, 90 (1), 013903-013903.

(18) Wang, Y.; Jing, W.; Tao, N.; Wang, H. Probing Single-Molecule Binding Event by the Dynamic Counting and Mapping of Individual Nanoparticles. ACS Sensors 2021, 6 (2), 523-529.

(19) Wulf, M.; de Hoogh, A.; Rotenberg, N.; Kuipers, L. Ultrafast plasmonics on gold nanowires: confinement, dispersion, and pulse propagation. ACS Photonics 2014, 1 (11), 1173-1180.

(20) Gramotnev, D. K.; Bozhevolnyi, S. I. Nanofocusing of electromagnetic radiation. Nat. Photonics 2014, 8 (1), 13-22.

(21) Zenin, V. A.; Andryieuski, A.; Malureanu, R.; Radko, I. P.; Volkov, V. S.; Gramotnev, D. K.; Lavrinenko, A. V.; Bozhevolnyi, S. I. Boosting Local Field Enhancement by on-Chip Nanofocusing and Impedance-Matched Plasmonic Antennas. Nano Lett. 2015, 15 (12), $8148-8154$.

(22) Zenin, V. A.; Malureanu, R.; Radko, I. P.; Lavrinenko, A. V.; Bozhevolnyi, S. I. Near-field characterization of bound plasmonic 
modes in metal strip waveguides. Opt. Express 2016, 24 (5), 45824590.

(23) Lozan, O.; Sundararaman, R.; Ea-Kim, B.; Rampnoux, J.-M.; Narang, P.; Dilhaire, S.; Lalanne, P. Increased rise time of electron temperature during adiabatic plasmon focusing. Nat. Commun. 2017, 8 (1), 1656.

(24) Verhagen, E.; Spasenović, M.; Polman, A.; Kuipers, L. Nanowire Plasmon Excitation by Adiabatic Mode Transformation. Phys. Rev. Lett. 2009, 102 (20), 203904.

(25) Schuller, J. A.; Barnard, E. S.; Cai, W. S.; Jun, Y. C.; White, J. S.; Brongersma, M. L. Plasmonics for extreme light concentration and manipulation. Nat. Mater. 2010, 9 (3), 193-204.

(26) Verhagen, E.; Kuipers, L.; Polman, A. Enhanced Nonlinear Optical Effects with a Tapered Plasmonic Waveguide. Nano Lett. 2007, 7 (2), 334-337.

(27) de Hoogh, A.; Opheij, A.; Wulf, M.; Rotenberg, N.; Kuipers, L. Harmonics Generation by Surface Plasmon Polaritons on Single Nanowires. ACS Photonics 2016, 3 (8), 1446-1452.

(28) Parzefall, M.; Novotny, L. Light at the End of the Tunnel. ACS Photonics 2018, 5 (11), 4195-4202.

(29) Weeber, J.-C.; Lacroute, Y.; Dereux, A. Optical near-field distributions of surface plasmon waveguide modes. Phys. Rev. B: Condens. Matter Mater. Phys. 2003, 68 (11), 115401.

(30) Zia, R.; Schuller, J. A.; Brongersma, M. L. Near-field characterization of guided polariton propagation and cutoff in surface plasmon waveguides. Phys. Rev. B: Condens. Matter Mater. Phys. 2006, 74 (16), 165415.

(31) Zia, R.; Selker, M. D.; Brongersma, M. L. Leaky and bound modes of surface plasmon waveguides. Phys. Rev. B: Condens. Matter Mater. Phys. 2005, 71 (16), 165431.

(32) Lamprecht, B.; Krenn, J. R.; Schider, G.; Ditlbacher, H.; Salerno, M.; Felidj, N.; Leitner, A.; Aussenegg, F. R.; Weeber, J. C. Surface plasmon propagation in microscale metal stripes. Appl. Phys. Lett. 2001, 79 (1), 51-53.

(33) Zia, R.; Chandran, A.; Brongersma, M. L. Dielectric waveguide model for guided surface polaritons. Opt. Lett. 2005, 30 (12), 14731475.

(34) Grandidier, J.; des Francs, G. C.; Markey, L.; Bouhelier, A.; Massenot, S.; Weeber, J. C.; Dereux, A. Dielectric-loaded surface plasmon polariton waveguides on a finite-width metal strip. Appl. Phys. Lett. 2010, 96 (6), 063105.

(35) Berthelot, J.; Tantussi, F.; Rai, P.; Colas des Francs, G.; Weeber, J.-C.; Dereux, A.; Fuso, F.; Allegrini, M.; Bouhelier, A. Determinant role of the edges in defining surface plasmon propagation in stripe waveguides and tapered concentrators. J. Opt. Soc. Am. B 2012, 29 (2), 226-231.

(36) Berthelot, J.; Bouhelier, A.; des Francs, G. C.; Weeber, J.-C.; Dereux, A. Excitation of a one-dimensional evanescent wave by conical edge diffraction of surface plasmon. Opt. Express 2011, 19 (6), 5303-5312.

(37) Wei, H.; Pan, D.; Zhang, S.; Li, Z.; Li, Q.; Liu, N.; Wang, W.; $\mathrm{Xu}, \mathrm{H}$. Plasmon Waveguiding in Nanowires. Chem. Rev. 2018, 118 (6), 2882-2926.

(38) Makarenko, K. S.; Hoang, T. X.; Duffin, T. J.; Radulescu, A.; Kalathingal, V.; Lezec, H. J.; Chu, H.-S.; Nijhuis, C. A. Efficient Surface Plasmon Polariton Excitation and Control over Outcoupling Mechanisms in Metal-Insulator-Metal Tunneling Junctions. Adv. Sci. 2020, 7 (8), 1900291.

(39) Radulescu, A.; Makarenko, K. S.; Hoang, T. X.; Kalathingal, V.; Duffin, T. J.; Chu, H.-S.; Nijhuis, C. A. Geometric control over surface plasmon polariton out-coupling pathways in metal-insulatormetal tunnel junctions. Opt. Express 2021, 29 (8), 11987-12000.

(40) Zhang, C.; Hugonin, J.-P.; Coutrot, A.-L.; Sauvan, C.; Marquier, F.; Greffet, J.-J. Antenna surface plasmon emission by inelastic tunneling. Nat. Commun. 2019, 10 (1), 4949.

(41) Duffin, T. J.; Kalathingal, V.; Radulescu, A.; Li, C.; Pennycook, S. J.; Nijhuis, C. A. Cavity Plasmonics in Tunnel Junctions: Outcoupling and the Role of Surface Roughness. Phys. Rev. Appl. 2020, 14 (4), 044021.
(42) Beane, G.; Brown, B. S.; Devkota, T.; Hartland, G. V. LightLike Group Velocities and Long Lifetimes for Leaky Surface Plasmon Polaritons in Noble Metal Nanostripes. J. Phys. Chem. C 2019, 123 (25), 15729-15737.

(43) Yang, H.; Qiu, M.; Li, Q. Identification and control of multiple leaky plasmon modes in silver nanowires. Laser Photonics Rev. 2016, 10 (2), 278-286.

(44) Song, M.; Dellinger, J.; Demichel, O.; Buret, M.; Colas Des Francs, G.; Zhang, D.; Dujardin, E.; Bouhelier, A. Selective excitation of surface plasmon modes propagating in Ag nanowires. Opt. Express 2017, 25 (8), 9138-9149.

(45) Lin, Y.; Hoang, T. X.; Chu, H.-S.; Nijhuis, C. A. Directional launching of surface plasmon polaritons by electrically driven aperiodic groove array reflectors. Nanophotonics 2021, 10 (3), 1145-1154.

(46) Du, W.; Wang, T.; Chu, H.-S.; Nijhuis, C. A. Highly efficient on-chip direct electronic-plasmonic transducers. Nat. Photonics 2017, 11 (10), 623-627.

(47) Takahara, J.; Yamagishi, S.; Taki, H.; Morimoto, A.; Kobayashi, $\mathrm{T}$. Guiding of a one-dimensional optical beam with nanometer diameter. Opt. Lett. 1997, 22 (7), 475-477.

(48) Kurdi, B. N.; Hall, D. G. Analysis of electromagnetic modes of aluminum-aluminum oxide-gold tunnel junctions. Phys. Rev. B: Condens. Matter Mater. Phys. 1986, 34 (6), 3980-3987.

(49) Yuan, L.; Jiang, L.; Nijhuis, C. A. The Drive Force of Electrical Breakdown of Large-Area Molecular Tunnel Junctions. Adv. Funct. Mater. 2018, 28 (28), 1801710.

(50) Simmons, J. G. Intrinsic Fields in Thin Insulating Films between Dissimilar Electrodes. Phys. Rev. Lett. 1963, 10 (1), 10-12.

(51) Segal, D.; Nitzan, A. Heating in current carrying molecular junctions. J. Chem. Phys. 2002, 117 (8), 3915-3927.

(52) Lumerical Inc., www.lumerical.com/.

(53) Olmon, R. L.; Slovick, B.; Johnson, T. W.; Shelton, D.; Oh, S.H.; Boreman, G. D.; Raschke, M. B. Optical dielectric function of gold. Phys. Rev. B: Condens. Matter Mater. Phys. 2012, 86 (23), 235147. 